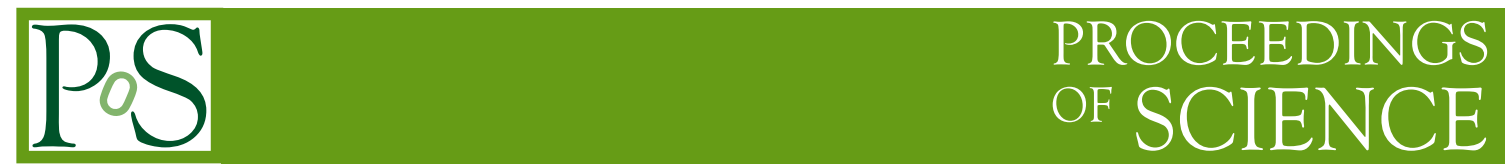

\title{
Soft wall model with arbitrary intercept
}

\author{
S. S. Afonin ${ }^{* \dagger}$ \\ Saint-Petersburg State University, Russia \\ E-mail: afonin24@mail.ru
}

\begin{abstract}
An exactly solvable generalization of the soft wall holographic model for the vector mesons which contains an additional free parameter is proposed. This parameter provides an arbitrary intercept in the Regge like spectrum of radial excitations and leads to a modification of asymptotic expansion of the vector correlator at large momentum. The matching to the Operator Product Expansion from QCD allows to make a strong restriction on possible values of the new parameter. Some phenomenological fits are presented.
\end{abstract}

Xth Quark Confinement and the Hadron Spectrum,

October 8-12, 2012

TUM Campus Garching, Munich, Germany

\footnotetext{
*Speaker.

${ }^{\dagger}$ The work is supported by the Dynasty Foundation.
} 
The Soft Wall (SW) holographic model for the strongly coupled QCD [1] has provided a seminal theoretical setup for many applications. A natural question arises whether it is possible to generalize this model. Unfortunately, the existing extensions and modifications spoil its main attractive feature consisting in the simplicity and exact solvability. In the present report, we discuss a simple generalization of the SW model which preserves its properties and is exactly solvable [2]. In the framework of our generalization, the intercept of the linear mass spectrum is not fixed as in the original model [1], instead it can take an arbitrary value. This feature modifies the structure of the vector correlator. The matching to the operator product expansion allows to impose hard restrictions on the intercept which follow from QCD.

In short, if we wish to have a linear vector mass spectrum with an arbitrary intercept,

$$
m_{n}^{2}=4 a(n+1+b)
$$

the action of the generalized SW model must be [2]

$$
S=\int d^{4} x d z \sqrt{g} e^{-a z^{2}} U^{2}\left(b, 0 ; a z^{2}\right)\left(-\frac{1}{4 g_{5}^{2}} F_{M N} F^{M N}\right),
$$

where $U$ is the Tricomi confluent hypergeometric function.

The vector correlator calculated using the action (2) reads

$$
\Pi_{V}\left(q^{2}\right)=\frac{R}{2 g_{5}^{2}}\left\{-\psi\left(1+b-\tilde{q}^{2}\right)+\frac{b}{\tilde{q}^{2}}\left[\psi\left(1+b-\tilde{q}^{2}\right)-\psi(1+b)\right]\right\} .
$$

Here $\psi$ denotes the digamma function and $\tilde{q} \equiv q^{2} / 4 a$. The spectral representation for the correlator under consideration is,

$$
\Pi_{V}\left(q^{2}\right)=-\sum_{n=0}^{\infty} \frac{F_{n}^{2}}{q^{2}-4 a(n+1+b)}, \quad F_{n}^{2}=\frac{2 a R}{g_{5}^{2}}\left(1-\frac{b}{4 a(n+1+b)}\right) .
$$

The poles of the correlator yield the mass spectrum (1). At $b \neq 0$ the residues (they determine the electromagnetic decay width, $\Gamma_{\mathrm{em}} \sim F^{2}$ ) acquire a dependence on $n$. Choosing $b<0$ this new feature allows to mimic a kind of the vector dominance: The lightest vector state possesses the largest value of residue.

The expansion of the correlator (3) at large Euclidean momentum $Q^{2}=-q^{2}$ leads to

$$
\begin{aligned}
\left.\Pi_{V}\right|_{Q^{2} \rightarrow \infty}=\frac{R}{2 g_{5}^{2}}\left\{\log \left(\frac{4 a}{Q^{2}}\right)\right. & -\frac{4 a}{Q^{2}}\left[\frac{1}{2}+b\left(\log \left(\frac{4 a}{Q^{2}}\right)+1-\psi(1+b)\right)\right] \\
+ & \left.\frac{1}{2}\left(\frac{4 a}{Q^{2}}\right)^{2}\left(\frac{1}{6}-b^{2}\right)+\frac{1}{6}\left(\frac{4 a}{Q^{2}}\right)^{3} b\left(b^{2}-\frac{1}{2}\right)+\mathscr{O}\left(Q^{-8}\right)\right\} .
\end{aligned}
$$

The expansion (5) can be matched to the Operator Product Expansion (OPE) for the vector twopoint correlator [3],

$$
\Pi_{V}^{(\mathrm{OPE})}=\frac{N_{c}}{24 \pi^{2}} \log \left(\frac{\mu_{\mathrm{ren}}^{2}}{Q^{2}}\right)+\frac{\alpha_{s}}{24 \pi} \frac{\left\langle G^{2}\right\rangle}{Q^{4}}-\frac{14 \pi \alpha_{s}}{9} \frac{\langle\bar{q} q\rangle^{2}}{Q^{6}}+\mathscr{O}\left(Q^{-8}\right) .
$$


The matching of coefficients in front of the leading logarithm provides the standard normalization factor, $\frac{R}{g_{5}^{2}}=\frac{N_{c}}{12 \pi^{2}}$.

In principle, the free parameters of the model - the slope $4 a$ and the (dimensionless) contribution to the intercept $b-$ can be fixed by matching the $\mathscr{O}\left(Q^{-4}\right)$ and $\mathscr{O}\left(Q^{-6}\right)$ terms. For the typical phenomenological values of the gluon and quark condensates, $\frac{\alpha_{s}}{\pi}\left\langle G^{2}\right\rangle=(360 \mathrm{MeV})^{4}$ and $\langle\bar{q} q\rangle=-(235 \mathrm{MeV})^{3}$, one obtains $4 a=(905 \mathrm{MeV})^{2}$ and $b=0.046$. Taking into account the qualitative character of the model, these estimates look reasonable.

A more conservative point of view on the $\mathscr{O}\left(Q^{-6}\right)$ term would be to consider it as non-reliable for numerical fits because of the asymptotic nature of the expansion. Within the standard SW model, $b=0$, taking a typical phenomenological value for the slope of meson trajectories [4], $4 a \approx(1.1 \mathrm{GeV})^{2}$, the matching of $\mathscr{O}\left(Q^{-4}\right)$ terms in the expansions (5) and (6) predicts a unrealistically large value for the gluon condensate, $\frac{\alpha_{s}}{\pi}\left\langle G^{2}\right\rangle \approx(440 \mathrm{MeV})^{4}$. The parameter $b$ allows to remove this drawback: It can be fixed from the condition to have a realistic gluon condensate in the expansions (5), $b^{2}=\frac{1}{6}-\frac{2 \pi^{2} \frac{\alpha_{s}}{\pi}\left\langle G^{2}\right\rangle}{N_{c}(4 a)^{2}}$. Substituting the physical values for the slope and gluon condensate to the condition (7), we arrive at the estimate $|b| \approx 0.3$.

The positivity of the $\mathscr{O}\left(Q^{-4}\right)$ term in the OPE leads to the constraint $|b|<1 / \sqrt{6}$ in the expansion (5). In addition, since this term is universal in the OPE for the vector and axial-vector channels [3] and depends quadratically on $b$, one has an intriguing possibility for a self-consistent mass splitting between the vector $(V)$ and axial $(A)$ states: The corresponding spectra have universal absolute value of $b$ but opposite sign, $m_{V, A}^{2}(n)=4 a(n+1 \mp|b|)$.

The nonzero values of $b$ generate the $\mathscr{O}\left(Q^{-6}\right)$ term in the OPE and this represents a new feature in comparison with the usual SW model. In the latter, the term $\mathscr{O}\left(Q^{-6}\right)$ is absent because the intercept (in units of the slope) is equal to unity. It is well known [5] that this is one of values of intercept at which the term $\mathscr{O}\left(Q^{-6}\right)$ disappears in the OPE of the two-point correlators saturated by the narrow resonances with linearly rising spectrum. It is interesting to note that in the model of the previous paragraph, the $\mathscr{O}\left(Q^{-6}\right)$ term in the $V$ and $A$ correlators differ by sign only (see Eq. (5)). This is somewhat close to the real OPE where these terms differ by the factor $-\frac{7}{11}[3]$.

In summary, we have shown how to introduce an arbitrary intercept to the linear spectrum of the SW model in a self-consistent way. The obtained generalization of the SW model remains exactly solvable. The resulting freedom in the choice of the intercept entails a sizeable modification of the two-point correlator, specifically a contribution related to the quark condensate is generated and the residues of meson poles cease to be universal for all states.

\section{References}

[1] A. Karch, E. Katz, D. T. Son and M. A. Stephanov, Phys. Rev. D74, 015005 (2006).

[2] S. S. Afonin, arXiv:1210.5210 [hep-ph].

[3] M. A. Shifman, A. I. Vainstein and V. I. Zakharov, Nucl. Phys. B147, 385, 448 (1979).

[4] A. V. Anisovich, V. V. Anisovich and A. V. Sarantsev, Phys. Rev. D62, 051502(R) (2000); D. V. Bugg, Phys. Rept. 397, 257 (2004).

[5] S. S. Afonin, Phys. Lett. B576, 122 (2003); S. S. Afonin, A. A. Andrianov, V. A. Andrianov and D. Espriu, JHEP 0404, 039 (2004); S. S. Afonin and D. Espriu, JHEP 0609, 047 (2006). 